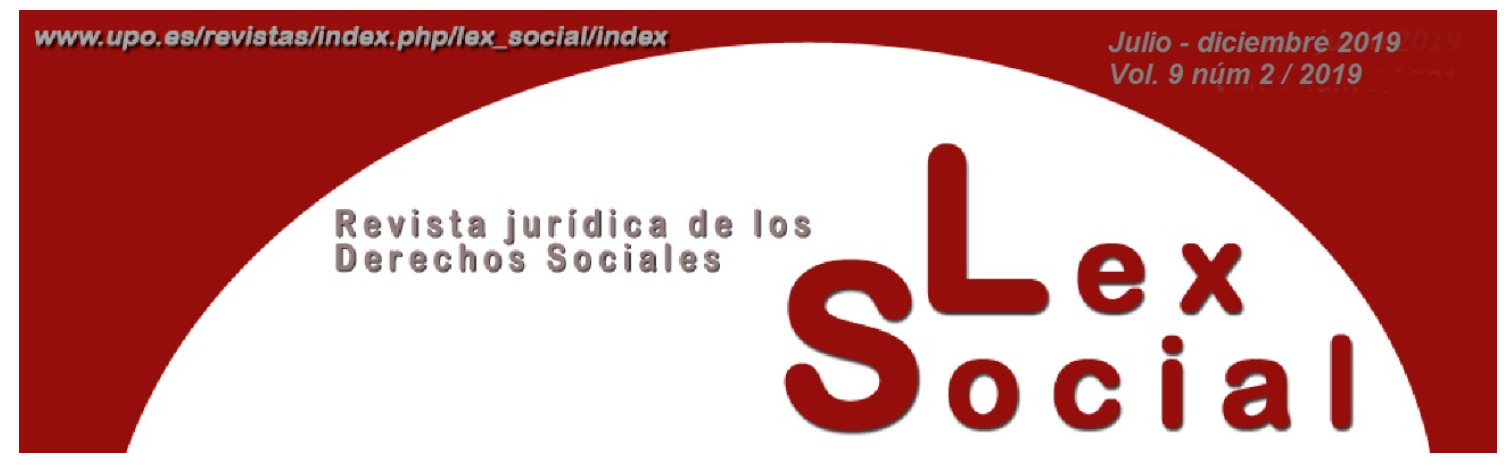

\title{
SERVICIO DOMÉSTICO Y ACTIVIDAD DE CUIDADOS EN EL HOGAR: LA ENCRUCIJADA DESDE LO PRIVADO Y LO PÚBLICO
}

\section{DOMESTIC SERVICE AND CARE: THE CROSSROADS FROM THE PRIVATE AND THE PUBLIC STANDPOINT}

IRENE LEBRUSÁN MURILLO

Investigadora posdoctoral, Harvard Law School

Universidad de Harvard

Artículo recibido el 26 de abril de 2019

Artículo aceptado el 27 de mayo de 2019

\section{RESUMEN}

Los cambios sociales y demográficos propios de las sociedades actuales dan paso a la necesidad de reconfigurar el funcionamiento habitual de los cuidados: las demandas aumentan y se diversifican, mientras que disminuye la capacidad real de su cobertura por parte de las familias. Las respuestas desde el Estado ante este desequilibrio son claramente insuficientes, propias de un Estado asistencialista que desplaza la responsabilidad del cuidado al ámbito familiar y que principalmente asumen las mujeres. Ante esta tesitura, las familias recurren a la externalización de los cuidados. Como estrategia privada destaca especialmente el recurso a la contratación de servicio doméstico, que deja de asociarse a la posición social. Si bien la contratación de personas para el cuidado implica una forma de reconocer económicamente labores que nunca han sido consideradas productivas, la regulación normativa del sector es insuficiente y refleja un claro desinterés jurídico y político. Estamos ante una encrucijada de los cuidados que no encuentra 
solución ni desde lo privado ni desde lo público: en primer lugar, la familia no puede dar respuesta a la necesidad de cuidado. En segundo lugar, el Estado no aporta soluciones públicas suficientes. Por último, la regulación del servicio doméstico, que impone una desigualdad clara frente al resto de trabajadores del régimen general, sume a trabajadoras y por lo tanto a los demandantes de cuidado en una situación de vulnerabilidad.

Palabras Clave: cuidados; servicio doméstico; Estado de bienestar; familia; estrategias

\begin{abstract}
Social and demographic changes lead to the need to reconfigure the usual functioning of care: while care demands increase and diversify, the actual capacity of their coverage by families decreases. The response from the State to this imbalance is clearly insufficient, typical of a welfare state that shifts the responsibility of care to the family and that is mainly assumed by women. Given this situation, families resort to the outsourcing of care. As a private strategy, the use of domestic service, which is no longer associated with social position, is particularly highlighted. Although the hiring of people for care implies a way of economically recognizing tasks that have never been considered productive, the regulation of the sector is insufficient and reflects a clear legal and political disinterest. So, we are at a crossroads where neither familiar or state care solutions are sufficient. First, the family cannot cover the care need. Secondly, the State does not provide adequate and sufficient public care alternatives. Overall, lack of regulation for domestic workers clearly differentiates these workers from others from a legal standpoint, causing a double vulnerability in workers and in the receptors of care.
\end{abstract}

KEYWORDS: care; domestic service; welfare state; family; strategies

SUMARIO

1. INTRODUCCIÓN

2. EL CUIDADO Y SU ORGANIZACIÓN

3. LOS CAMBIOS SOCIALES Y LA DENOMINADA CRISIS DE CUIDADOS

4. EL ESTADO DE BIENESTAR Y LOS CUIDADOS: ALCANCES LIMITADOS Y RECORTES

5. DE LA DESVALORIZACIÓN SOCIAL A LA LABORAL: EL SERVICIO DOMÉSTICO 


\section{REFLEXIONES FINALES.}

\section{INTRODUCCION}

El cuidado ha sido considerado tradicionalmente una cuestión privada, que recaía principalmente sobre las mujeres. Sin embargo, en los últimos años se producen una serie de cambios tanto sociales como demográficos que alteran las relaciones de cuidados y ponen en duda la continuidad del sistema tradicional de provisión. No solo la demanda ha aumentado, sino que la capacidad de cobertura ha disminuido, por los motivos que veremos.

Tal situación de desequilibrio y la escasa respuesta por parte del Estado del bienestar, que se define como un sistema asistencialista e incompleto, hacen que estemos ante una encrucijada de los cuidados, que no encuentra solución plena ni desde lo privado ni desde lo público. Como consecuencia, es necesario que estos cuidados se mercantilicen y sean cubiertos fuera del ámbito familiar, de modo que nuevos actores entran en la ecuación familia-cuidado. La estrategia privada que más aumenta es la contratación de servicio doméstico.

La externalización de los cuidados en realidad no es un fenómeno tan novedoso (recordemos a las amas de cría) pero hasta mediados del s. XX estaba relacionada con el estatus social de aquellas familias que se lo podían permitir. Es decir, la externalización de los cuidados y otras labores domésticas se consideraban una forma de demostrar la posición social ${ }^{1}$ más que una estrategia de conciliación. En la actualidad, el recurso al servicio doméstico como forma de externalizar los cuidados ha dejado de ser algo propio de familias acomodadas, pasando a ser parte de las prácticas habituales de familias de clase media e incluso de sectores con recursos económicos limitados ${ }^{2}$. Por lo tanto, los cuidados a familiares, específicamente mayores y niños, tradicionalmente escenario asociado a la mujer, se desplazan fuera del espacio familiar. Esta situación, paradójicamente, no resulta en una "liberación femenina” en tanto que el rol de los cuidados pasan a manos de otras mujeres asalariadas, generalmente inmigrantes.

Como consecuencia, se ha producido un enorme crecimiento del sector del servicio doméstico durante los últimos años, con efectos tanto sobre personas ocupadas como en la propia economía. De hecho, el empleo doméstico en España, representa en torno al 5\% de la ocupación, una cifra bastante alta si se compara con la media de países desarrollados donde apenas alcanza el $1 \%^{3}$. No obstante, las condiciones laborales del sector, diferenciadas del resto de regímenes, reflejan simbólicamente la

\footnotetext{
${ }^{1}$ SANTOS Y VALENCIA, 2011.

2 LEBRUSÁN Y CÁCERES, 2017.

${ }^{3}$ ARANGO, DÍAZ Y MOUALHI, 2013.
} 
minusvalorización social de los cuidados, dejando a las trabajadoras (en femenino, ya que en su gran mayoría son mujeres) en situación de desprotección y por lo tanto, añadiendo nuevas dimensiones de precariedad a los cuidados.

Esta artículo revisa el propio concepto del cuidado, distinguiendo el cuidado formal del informal, a fin de conocer la estructura de organización. A continuación ahonda sobre las cuestiones sociales y demográficas que dan lugar a lo que algunas autoras han denominado "la crisis de los cuidados" para pasar a analizar brevemente los recursos aportados por parte del Estado del bienestar. Seguidamente se analiza la normativa laboral del servicio doméstico para comprender las dificultades que enfrenta este sector y por último se exponen unas reflexiones finales.

\section{EL CUIDADO Y SU ORGANIZACIÓN}

Por cuidado se entiende el apoyo o asistencia que se proporciona a las personas que lo necesitan. Los potenciales demandantes serían niños y niñas, personas mayores y personas dependientes en general. Según la concepción teórica del “cuidado social”, el cuidado infantil y las personas mayores frágiles formarían parte de una relación social horizontal y recíproca, emocional, física y de mediación que implica la asistencia práctica y vigilancia ${ }^{4}$. No obstante, todos podemos ser receptores de labores de cuidado en distintos momentos de nuestra vida, incluso aunque no quepamos a priori en la definición de dependientes o como parte de esa relación social horizontal referida de cuidado social. Sin precisar asistencia podemos ser receptores a diario de labores de cuidado que hacen que nuestra vida cotidiana sea más fácil. Sin embargo, estas labores que, bien son imprescindibles para la continuidad de la vida o bien, tanto facilitan el día a día, han sido tradicionalmente desprestigiadas y consideradas completamente al margen de las labores productivas.

Dentro del cuidado distinguiríamos el cuidado informal del cuidado formal. Mientras que el cuidado informal corresponde principalmente al ámbito familiar o privado, el cuidado formal refiere aquél que se externaliza, y en el que aparecen como agentes intervinientes el Estado y el mercado. Ambos tipos de cuidado se vertebran en torno a un eje marcado por las relaciones de género y son estructurados y contextualizadas por la forma, tamaño y la potencial intervención del Estado del bienestar.

Dentro del cuidado formal entenderíamos aquellas formas de cuidado por las que se recibe una remuneración, que están reguladas en función de las condiciones fijadas por un marco jurídico y que por lo tanto quedan ajustadas a reglas y normativas específicas establecidas por el Estado. En este sentido, supone el reconocimiento económico de labores que se han considerado libres de coste. Además, al menos teóricamente, estas labores de cuidado se registran oficialmente y están sujetas al pago de una serie de

\footnotetext{
${ }^{4}$ FLAQUER, PFAU-EFFINGER Y ARTIAGA, 2014.
} 
impuestos y de costes laborales. No obstante, distintas investigaciones han señalado la gran presencia de trabajo informal o en situación de economía sumergida en el sector de los cuidados ${ }^{5}$.

La diferencia clave entre cuidado formal e informal estriba en que, si bien todas las formas de cuidado suponen trabajo, solo los cuidados formales pueden considerarse empleo.

Al respecto de esta consideración cabe reflexionar sobre cómo se diferencian el empleo y el trabajo, pero especialmente sobre el impacto social que esto tiene sobre las personas que emiten el cuidado. El trabajo es un concepto ambiguo que refleja un conflicto social en torno al reconocimiento y legitimación de las actividades humanas en términos de productividad $^{6}$. Todas las formas de trabajo estructuran nuestra vida, pero el empleo señala nuestro posicionamiento en la sociedad (como parte o no de la estructura productiva) y nos diferencia en clave de acceso a ciertos derechos que marca el Estado de bienestar. Es decir, el gran problema desde el punto de vista de la inequidad social y el acceso a derechos es que, mientras que el empleo, además de contar con una retribución, genera derechos dentro del Sistema de bienestar (por ejemplo, derecho a pensión por jubilación) y forma parte de la esfera pública, el trabajo de cuidados informal no genera derechos sociales.

En general y tradicionalmente, los cuidados se consideran propios de la esfera privada, siendo competencia principalmente de las familias. Esto es en parte resultado de una preferencia mayoritaria por el cuidado informal exclusivo en España, aunque el rol de los servicios públicos y/o privados se demanda y valora de una forma más positiva que en el pasado ${ }^{7}$.

En realidad cuando hablamos de familia y cuidados, hablamos de una responsabilidad que recae de manera predominante y casi exclusiva sobre la mujer. Como señalan algunas autoras, la responsabilidad del cuidado de las personas mayores recae sobre la mujer o las hijas preferentemente ${ }^{8}$ o sobre las abuelas en el caso del cuidado de niños ${ }^{9}$. Los motivos tras esta organización de los cuidados responden a cuestiones tanto de socialización como de la división sexual del trabajo, con fuerte influencia del mercado laboral y con el tipo de protección social ofertado por el Estado.

No obstante, en los últimos años se producen una serie de cambios en las estructuras demográficas y principalmente, un cambio en la organización de los roles tradicionalmente desarrollados por la mujer. Esto lleva a un desequilibrio en torno a los

\footnotetext{
${ }^{5}$ LEBRUSÁN Y CÁCERES, 2017; GARCÍA, SANTOS Y VALENCIA, 2014.

${ }^{6}$ SERRANO, MORENO Y CRESPO, 2001.

${ }^{7}$ FERNÁNDEZ CARRO, 2018.

${ }^{8}$ FERNÁNDEZ CARRO, 2018.

${ }^{9}$ TOBÍO, 2013.
} 
cuidados y su organización, que hace que estemos ante lo que algunos autores ${ }^{10}$ han denominado la crisis de los cuidados. En este sentido cabría señalar que, mientras que se reconoce el derecho a recibir cuidados ${ }^{11}$, no se reconoce del mismo modo el derecho a la libre elección sobre cuidar o no cuidar ${ }^{12}$.

\section{LOS CAMBIOS SOCIALES Y LA DENOMINADA CRISIS DE CUIDADOS}

Bajo la idea de "crisis de los cuidados" 13 se esconde la incapacidad de la familia nuclear y de la familia extendida para hacer frente a una demanda de cuidados en aumento y ante la que el Estado del bienestar no parece plantear alternativas efectivas.

Para entender cómo se conforma este "desequilibrio" en los cuidados hemos de referir diversos procesos: los que conciernen a la mujer y su comportamiento en el mercado laboral; el cambio demográfico, que implica una mayor longevidad y una disminución en las cohortes de potenciales cuidadoras; cambios sociales asociados a esta nueva longevidad (aging in place); cambios relacionados con las pautas de convivencia, entre otros que conciernen a la mayor exigencia del mercado laboral.

En lo que respecta a la mujer, se produce no solo la incorporación al mercado laboral de forma generalizada (España cerró 2018 con 8.826.470 afiliadas a la Seguridad Social y lidera la creación de empleo femenino en la Eurozona), que no es algo tan novedoso ${ }^{14}$, sino que esta incorporación se acompaña de su normalización social ${ }^{15}$. Además, en conjunción con esta normalización se producen una serie de cambios en su comportamiento y relación con el mercado laboral, así como un cambio en sus deseos profesionales. Los más relevantes de estos cambios serían su permanencia en el trabajo tras la maternidad ${ }^{16} \mathrm{y}$ la disociación entre matrimonio y abandono del puesto laboral que caracterizaba el comportamiento de generaciones anteriores ${ }^{17}$. Esta afirmación no obvia la continuidad de la segregación en el mercado de trabajo tanto a nivel vertical (acceso a posiciones directivas) como horizontal (distinción entre puestos asignados tradicionalmente a posiciones femeninas y masculinas) además de otras “penalizaciones” como la brecha salarial entre ambos géneros, el alto porcentaje de

\footnotetext{
${ }^{10}$ OROZCO, 2007.

${ }^{11} \mathrm{Al}$ respecto de esta concepción y sus implicaciones desde un punto de vista del derecho social, véase HOLGADO, 2019.

12 PAZOS, 2013. TOBÍO (2013) señala lo contrario, si bien el derecho a no cuidar estaría reservado a los hombres.

${ }^{13}$ OROZCO, 2007.

14 En España, hasta los años setenta la actividad laboral femenina estaba fuertemente asociada a coyunturas económicas o bélicas a través de salidas al mercado de trabajo y repliegues posteriores al hogar. Sin embargo, en casi todos los países desarrollados las tasas de actividad femenina aumentaron entre 1973 y 1979, años de recesión económica (TOBÍO, 2001)

15 TOBÍO, 2013

${ }^{16}$ Aunque esto ha sido y continua siendo fuente de discriminación en el ámbito laboral (SELMA, 2019).

${ }^{17}$ Véase LEBRUSÁN, 2017.
} 
contratos a tiempo parcial, y la difícil conciliación temporal entre la doble jornada ${ }^{18}$. Tampoco hay que olvidar que casi la mitad de las mujeres en edad activa (16-64 años) no están en el mercado de trabajo ${ }^{19}$ y se dedican a las tareas del hogar y los cuidados. Es decir, trabajan pero no están empleadas, lo que significa que no cotizan y no generan derechos propios relacionados con esta contribución.

El mercado laboral penaliza a la mujer no solo por el desempeño de cuidados cuando son necesarios, sino por la posibilidad de que en algún momento deba realizarlos. Es decir, a la mujer se le presupone la labor de cuidados incluso antes de que estos sean demandados, y eso tiene un efecto claro en su relación con el mercado laboral y en su propia historia de cotización.

Además, a las consabidas complicaciones a las que se enfrenta la mujer madre trabajadora, se le suman las crecientes exigencias en cuanto a dedicación y disponibilidad de tiempo (tanto para hombres como para mujeres) de un mercado de trabajo globalizado ${ }^{20}$ que complica enormemente la conciliación laboral y familiar. A esto se suman los tiempos de desplazamiento al centro laboral, que cada vez son mayores en sociedades segregadas residencialmente. El tiempo disponible para el cuidado disminuye enormemente.

Otro aspecto a tener en cuenta para comprender esta sobrecarga de cuidados sería el cambio demográfico. Vivimos más, y aunque lo hacemos en mejores condiciones de salud, un mayor número de personas vive hasta edades más (mucho más) avanzadas: en 2016 Eurostat señala a las españolas como las más longevas de Europa, con 86,3 años. Con 80,5 años de esperanza de vida, los varones españoles están en séptimo lugar. Este aumento en la esperanza de vida provoca que, proporcionalmente, aumente la necesidad de cuidados en la vejez; aunque las condiciones en las que se vive la vejez han mejorado, cuando finalmente necesitamos los cuidados, los necesitamos durante más tiempo. Además se produce otro cambio referido a la vejez: las personas mayores quieren vivir independientes durante todo el tiempo posible, aunque ello requiera de un mayor despliegue de cuidados y diversificación de estrategias, ya sea con apoyo puntual, cuidados parciales u otros mecanismos que se fundamentan en el soporte social de familiares inmediatos ${ }^{21}$. Por otra parte, la mayor supervivencia de personas con discapacidad y enfermedades crónicas, aumentan la complejidad de la provisión de cuidado ${ }^{22}$.

\footnotetext{
18 SABATER, 2014.

${ }^{19}$ La tasa de actividad de las mujeres es del 53,08\%, frente al 64,45\% de los hombres, lo que indica una brecha de 11,37 puntos (MINISTERIO DE SEGURIDAD SOCIAL, 2019).

${ }^{20}$ OSO Y PARELLA, 2012.

${ }^{21}$ LEBRUSÁN, 2017.

${ }^{22}$ GARCÍA-CALVENTE ET AL., 2004.
} 
A esto se añade la reducción de cohortes en las generaciones que serían potenciales cuidadores, que hace que se reduzca el número de efectivos disponibles para el cuidado $^{23}$. Por decirlo de otro modo: más personas mayores viven durante más tiempo pero las generaciones que podrían realizar estos cuidados son de menor tamaño, por lo que nos encontramos que más personas necesitadas de cuidados cuentan con menor número de cuidadores potenciales. Podemos así encontrarnos a mujeres de edad avanzada que tienen que cuidar de ambos padres y de sus suegros, encontrándose desbordadas ante la demanda de cuidados. Se suele olvidar que pudiera ser que las condiciones físicas no respondan a la lógica esperada, y en ocasiones el potencial emisor de cuidados puede ser quien más los necesite ${ }^{24}$.

Esta barrera, puramente demográfica, resulta especialmente relevante a la hora de reflexionar sobre la configuración de los cuidados y la explicación de la situación de sobrecarga. También es útil para dejar de idealizar las relaciones de cuidado en el pasado. En resumidas cuentas, el supuesto equilibrio entre cuidadores y demandantes de cuidados que podía darse en el pasado ya no existe.

Por otra parte, se produce un cambio en la estructura de los hogares y en las pautas de convivencia que hacen que determinadas estrategias familiares sean más complejas de llevar a cabo. Además las reconfiguraciones familiares complejizan el desempeño de la solidaridad familiar ampliada.

No podemos hablar, sin embargo, de una desfamiliarización de los cuidados; el 80\% de los cuidados se producen dentro de la familia ${ }^{25}$. Las familias (y de nuevo insistimos, las mujeres) son quienes sostienen la mayor responsabilidad del cuidado ${ }^{26}$ e incluso de la familia extensa con especial protagonismo de las abuelas ${ }^{27}$ y no parece que esto vaya a cambiar próximamente, como demuestran algunas investigaciones ${ }^{28}$.

Ante este desequilibrio y la dificultad de conciliación que recae principalmente sobre la mujer, el Estado del bienestar no parece plantear alternativas efectivas. España se caracteriza por la ausencia de una cultura pública del cuidado que permita avanzar hacia nuevos modelos de organización que no sitúen siempre a la mujer como figura cuidados en el hogar ${ }^{29}$. ¿En qué consiste la cultura pública del cuidado por parte del Estado? ¿Cómo se comprenden desde el Estado las medidas públicas dirigidas específicamente a los cuidados?

\footnotetext{
${ }^{23}$ LEBRUSÁN, 2017; ELIZALDE, 2018.

${ }^{24} \mathrm{Al}$ respecto, véase LEBRUSÁN (2017).

${ }^{25}$ ELIZALDE, 2018.

${ }^{26}$ MARTÍNEZ BUJÁN, 2014; TOBÍO ET AL. 2010; ELIZALDE, 2018; FERNÁNDEZ CARRO, 2018.

27 TOBÍO, 2013.

${ }^{28}$ FERNÁNDEZ CARRO, 2018.

${ }^{29}$ OSO Y PARELLA, 2012.
} 


\section{EL ESTADO DE BIENESTAR Y LOS CUIDADOS: ALCANCES LIMITADOS Y RECORTES}

El Estado de bienestar responde a un concepto de modelo general del Estado y de organización social según la cual el gobierno provee de ciertos servicios o garantías sociales dirigidas a mejorar el bienestar social y la calidad de vida de la población. Dentro de sus intervenciones, las que afectan de manera más explícita a la calidad de vida de los ciudadanos son los servicios públicos (sanidad, educación, servicios sociales...), las transferencias sociales (pensiones), las intervenciones normativas (normas y sanciones) y las intervenciones públicas ${ }^{30}$.

La provisión de cuidados desde el Estado se orienta a la protección social de las familias, que consiste en proporcionar a las familias recursos para que puedan cumplir adecuadamente sus funciones. Estas funciones refieren principalmente la provisión de bienestar a sus miembros y la de socialización o educación de los hijos. Para estos fines los poderes públicos transfieren dinero a las familias, facilitan determinados servicios y tiempo disponible para el cuidado de los familiares dependientes ${ }^{31}$. Es decir, según MEIL, el Estado facilitaría recursos monetarios y recursos temporales. España parece abogar más por la primera opción, facilitando deducciones bajo la idea de un sobresalario que compensaría los costes de la maternidad para aquellas madres que siguen trabajando durante los primeros 3 años. Esta parece una medida limitada no solo en alcance sino en la propia conceptualización del cuidado, ya que tales deducciones no solventan las necesidades de cuidado de los menores.

La motivación que subyace a las transferencias familiares, como hemos señalado, es la de compensar los mayores costes y el menor nivel de renta per cápita de las familias, al tiempo que significa reconocer la rentabilidad social de las funciones familiares (tenencia de niños o cuidado de familiares dependientes) ${ }^{32}$. Es decir, se prima la cuestión económica de la familia y la supuesta pérdida adquisitiva Sin embargo, ni las transferencias ni los recursos temporales están lo suficientemente desarrollados como para permitir la conciliación familiar. Precisamente, los servicios públicos que en España están menos desarrollados son los servicios de ayuda las familias, como las escuelas de infancia de 0-3 años o los servicios domiciliarios a mayores y personas con discapacidad, entre otros. Este subdesarrollo pone en entredicho el discurso oficial del país que coloca a la familia en el centro de la sociedad, dificultando enormemente la conciliación familiar y con un sobrecoste en la calidad de vida y la salud, especialmente en el caso de la mujer ${ }^{33}$. En palabras de TOBÍO,

\footnotetext{
${ }^{30}$ NAVARRO Y QUIROGA, 2004.

${ }^{31}$ MEIL, 2005.

${ }^{32}$ MEIL 2005.

${ }^{33}$ NAVARRO Y QUIROGA, 2004.
} 
"El cambio de la familia parsoniana, basada en la división de roles de género, a la familia igualitaria en la que tanto el hombres como la mujer trabajan a cambio de una remuneración económica y, por tanto, proveen económicamente al hogar, no se ha acompañado en nuestro país, más que en escasa medida, de un apoyo público a través de políticas de conciliación”34.

Aunque los colegios ofertan la educación desde los 3 (recordemos que la educación obligatoria en España comienza a los 6) los horarios escolares se alejan de los horarios laborales. Por otra parte, y esta es una cuestión de especial relevancia, la intención de esta oferta es educativa, por lo que no pueden asumirse como formas publicas de facilitar la conciliación. No debemos confundir conciliación con educación, aunque ambas cuestiones puedan relacionarse. A nivel privado, algunas empresas ofertan guarderías y formas alternativas dirigidas al cuidado de niños de sus empleados. No sucede así con la atención a otros grupos demandantes de cuidado, y en cualquier caso, estamos ante programas puntuales y de carácter privado.

El otro extremo de la demanda de cuidado, el de las personas dependientes y las personas mayores, recibe aún menor atención pública. Surge una respuesta parcial en el año 2006, con la Ley de Promoción de la Autonomía Personal y Atención a las personas en situación de dependencia (Ley 39/2006 del 14 de diciembre). Apuntaba a ser un cuarto pilar del Estado del bienestar pero con los sucesivos recortes queda extremadamente reducida y no procura sino una respuesta parcial a estas necesidades de cuidado. Tampoco hay que olvidar que su objetivo era mejorar la calidad de vida de las personas con dificultades en las Actividades Básicas de la Vida Diaria (ABVD) ${ }^{35}$. Es decir, la finalidad de esta normativa es la promoción de la autonomía y de la permanencia en la vivienda, que en realidad es la alternativa más económica para el Estado $^{36}$.

Respecto a su evolución y a los impedimentos para dar respuesta a una necesidad social clara, el inicial baremo para reconocer la situación de dependencia (Real Decreto 504/2007) se deroga en 2011 (Real Decreto 174/2011 del 11 de Febrero), endureciendo los requisitos y por lo tanto, reduciendo la posibilidad de incorporaciones nuevas. Más tarde, el Real Decreto 1050/2013 impone nuevos cambios, regulando tres niveles de protección; uno mínimo con cargo a la Administración General del Estado, un segundo nivel acordado entre Estado y Comunidad Autónoma, y un nivel adicional sujeto a decisión de la Comunidad. En la práctica, esto implica una desigualdad territorial de la

\footnotetext{
34 TOBÍO, 2013: 17.

${ }^{35}$ La afectación de las ABVD pueden conducir a la institucionalización, siendo necesario el cuidado por parte de otras personas. Son, y se pierden por este orden: bañarse, vestirse, usar el retrete, movilizarse, ser continente y alimentarse.

${ }^{36}$ LEBRUSÁN, 2017.
} 
concesión de estas ayudas y provisión de servicios ${ }^{37}$ según el presupuesto de la Comunidad, además de la obligación de copagos por parte de la persona que recibe los cuidados.

Aunque prime el servicio, la ayuda puede ser otorgada en dinero o en servicios. La realidad es que, según la Asociación de directoras y gerentes de servicios sociales, en 2018 fallecieron diariamente más de 80 personas dependientes sin haber accedido a las prestaciones (de cualquier tipo) a las que tenían derecho ${ }^{38}$.

En definitiva, esta normativa contempla el apoyo económico a cuidadores no profesionales en el entorno familiar (parientes por consanguinidad, afinidad o adopción, hasta el tercer grado de parentesco) lo que supone un reconocimiento económico de la solidaridad familiar, pero de nuevo, ni estas ni las medidas en servicios se orientan a la conciliación. Por otra parte, en lo que respecta a la ayuda económica, los requisitos resultan especialmente poco acordes a la realidad de la aparición de la dependencia, al exigir una previsión de cuidado (convivencia desde al menos un año previo a la solicitud de la ayuda) que en la práctica no puede existir: en numerosas ocasiones necesidad de cuidado asociada a la dependencia no es previsible en el tiempo. Esto puede traducirse en una desprotección y aumento de la vulnerabilidad del hogar hasta que se genera derecho al cobro de la prestación o el disfrute del servicio.

En definitiva, y respecto a los servicios procurados desde el Estado, hay que tener en cuenta que "la construcción histórica de los sistemas públicos de bienestar social descansa sobre supuestos muy concretos acerca de la estructura y el papel que juega la familia en la provisión de bienestar a sus miembros ${ }^{39}$ ". Pero además se apoya fuertemente sobre la idea de familia nuclear tradicional, en la que el hombre es el ganapanes $^{40}$ y la esposa, dependiente económicamente, sería la responsable de dar respuesta a la necesidad de cuidados. Las medidas señaladas no concilian ni procuran cuidados, sino que refuerzan este modelo.

Cierto es que la atención y cuidados a los mayores y a la infancia se ha incrementado con el paso del tiempo ${ }^{41}$ pero indudablemente las provisiones desde el Estado siguen siendo insuficientes. Por otra parte, y como resultado de la crisis económica,

\footnotetext{
${ }^{37}$ Entre la oferta pública de la Red de Servicios Sociales (dependiente de cada Comunidad Autónoma) están: los Servicios de prevención de dependencia y de promoción de la autonomía personal; el Servicio de Teleasistencia; el Servicio de Ayuda a domicilio; el Servicio de Centro de Día y de Noche (distinguiendo para mayores y menores de 65 años); el Servicio de Atención Residencial.

${ }^{38}$ ASOCIACIÓN ESTATAL DE DIRECTORES Y GERENTES DE SERVICIOS SOCIALES, 2018. Disponible

en: https://www.directoressociales.com/images/Dec2019/INFO\%20GLOBAL\%20XIX\%20DICTAMEN\%20 V2-2.pdf. Consultado: abril 2019.

${ }^{39}$ MEIL, 2005: 19.

${ }^{40}$ VALIENTE, 1997.

${ }^{41}$ TOBÍO ET AL., 2010.
} 
“a partir de 2010, y a un ritmo acelerado en 2012, se produce una ruptura del proceso de construcción del Estado del bienestar, un retroceso de décadas tanto en las políticas de bienestar más consolidadas como en nuevos ámbitos como la conciliación o la igualdad de género" ${ }^{42}$.

Es decir, ante los recortes económicos, los avances en materia de cuidados desde el Estado, suelen ser los primeros en desaparecer, expresando el bajo valor social (y político) de los mismos.

Al respecto, como numerosos autores han señalado, "España especialmente se caracteriza por un sistema de bienestar poco desarrollado que se equilibra con el recurso a la figura familiar como factor constitutivo del sistema y como pilar básico de la estructura socioeconómica: lo que se conoce como familismo" ${ }^{43}$. Es decir, la solidaridad familiar se asume y se implementa como la forma de sustitución de las carencias del mercado y el Estado respecto a las necesidades sociales de cuidados. Pero este planteamiento de solidaridad familiar recae de forma predominante sobre la mujer, exigiendo un gran sacrificio personal y causando una serie de efectos no deseados sobre la calidad del cuidado y el bienestar personal de todos los componentes de esta ecuación. La forma en la que este sistema subsiste y funciona es mediante una mayor presión y exigencia sobre la mujer, que se enfrenta a dobles jornadas o a la elección entre su proyección laboral y las necesidades de cuidados. Se habla de la necesidad de protección de la familia, de la conciliación laboral y familiar, pero desde un enfoque en el que se sobreentiende que la mujer es la responsable de los cuidados y por tanto la que ha de realizar el esfuerzo.

Según plantean algunos autores ${ }^{44}$, sería la solidaridad intergeneracional característica de la sociedad española y su cobertura del bienestar familiar la que habría hecho que los gobiernos relajasen sus funciones de garantizar unos mínimos de protección a los colectivos vulnerables. Esta reducción del alcance del Estado se enmarcaría en una serie de estructuras clientelares y obsoletas propiciando la fragmentación de los sistemas de protección, que favorecen a algunos sectores y desamparan a otros ${ }^{45}$. En cualquier caso, lo cierto es que el cuidado sigue siendo una asignatura pendiente del sistema de bienestar español ${ }^{46}$.

Esta comprensión de los cuidados por parte del Estado da lugar a diversas ineficiencias y malfuncionamientos entre los que destacan la sobrecarga familiar y los problemas referidos que asume la mujer, pero también una mayor vulnerabilidad de los sectores

\footnotetext{
42 TOBÍO, 2013: 32.

43 MORENO Y MARI-KLOSE, 2016.

${ }^{44}$ MORENO Y MARI-KLOSE, 2013.

${ }^{45}$ MORENO Y MARI-KLOSE, 2013.

${ }^{46}$ FERNÁNDEZ CORDÓN, 2015; ELIZALDE, 2018.
} 
demandantes de cuidados. Aparecen fenómenos como "los niños de la llave", niños que pasan exceso de horas en soledad como resultado de la imposibilidad de conciliar la vida laboral y personal de los padres ${ }^{47}$.

Como señala ELIZALDE respecto de la atención a personas mayores, pero que indudablemente también aplica a otros colectivos demandantes de cuidados, la solución a la ecuación «necesidades de cuidados - disponibilidad de cuidadoras/es» necesita de nuevos elementos ${ }^{48}$.

Entre estos nuevos elementos, y en este contexto de crisis de los cuidados se produce la llegada de flujos migratorios conformados por mujeres que están dispuestas a trabajar en el sector del servicio doméstico y de los cuidados ${ }^{49}$. A este proceso se le ha denominado feminización de las migraciones ${ }^{50}$, referenciando el aumento de mujeres que realizan la migración de manera pionera dentro de sus familiares, no como acompañante o como parte de la reunificación. Estas mujeres plantean en su proyecto migratorio el servicio doméstico como un trabajo refugio, provisional. El sector de los cuidados se transforma así en un nicho laboral donde las mujeres migrantes encuentran la forma de acceder al mercado laboral, y de este modo acceder a la regularización administrativa $^{51}$.

Así, las necesidades no cubiertas y el desequilibrio entre la necesidad de cuidados, la escasa ayuda por parte del Estado y la sobrecarga de las familias (mujeres) hace que muchas familias españolas se conviertan en hogares empleadores de personal doméstico. No se trata de un proceso de privatización, en la medida en que la responsabilidad de los cuidados ya antes recaía en la esfera privada, si no de externalización. Este sería el resultado de la gestión neoliberal de la situación de sobrecarga, que deviene en la externalización e internacionalización de los cuidados ${ }^{52}$. El crecimiento del trabajo doméstico está además unido a políticas específicas de migración que han facilitado de forma implícita y explícita, el volumen de trabajadoras inmigrantes en este sector laboral ${ }^{53}$.

Cabría pensar que este exceso por parte de las familias encontrarían un reflejo en la valoración del servicio doméstico. Sin embargo, el del servicio doméstico es un sector históricamente desfavorecido, reflejo de cómo la desvalorización social de los cuidados se imprime incluso cuando las labores pasan a formar parte de la esfera del empleo.

${ }^{47}$ GIAMELLO Y CASTRO, 2017; 2018.

${ }^{48}$ ELIZALDE, 2018.

${ }^{49}$ MORENO Y BRUQUETAS, 2011.

${ }^{50}$ OROZCO, PAIEWONSKY Y GARCÍA, 2008.

${ }^{51}$ LEBRUSÁN Y CÁCERES, 2017; LEBRUSÁN, CÁCERES Y BREY, 2019.

${ }^{52}$ EZQUERRA, 2012.

${ }^{53}$ LEÓN, 2013. 


\section{DE LA DESVALORIZACIÓN SOCIAL A LA LABORAL: EL SERVICIO DOMÉSTICO}

La externalización de los cuidados en realidad no es un fenómeno tan novedoso (recordemos a las amas de cría) pero hasta mediados del s. XX estaba relacionada con el estatus social de aquellas familias que se lo podían permitir. Es decir, la externalización de los cuidados y otras labores domésticas se consideraban una forma de demostrar la posición social ${ }^{54}$ más que una estrategia de conciliación. En ese caso la externalización simboliza las tareas que no son deseables y que no se realizan cuando se puede elegir; los cuidados por lo tanto quedan asociados a un sector social, además de a un género concreto.

En la actualidad, el recurso al servicio doméstico como forma de externalizar los cuidados ha dejado de ser algo propio de familias acomodadas, pasando a ser parte de las prácticas habituales de familias de clase media e incluso de sectores con recursos económicos limitados ${ }^{55}$. Así, se ha producido un enorme crecimiento de los últimos años.

Lo anterior no se correlaciona en modo ninguno con una mayor atención normativa a este sector. Una breve revisión histórica de la su regulación demuestra el desinterés tanto social como legislativo que se imprime desde sus orígenes. Si bien en 1931 (Ley de Contrato de Trabajo de 21 de noviembre) se reconoce por vez primera a los ocupados del servicio doméstico dentro del concepto de trabajador y en 1944 se incluye como seguro específico dentro de los seguros profesionales, la Ley de Contrato de Trabajo de 1944 (Decretos de 26 de enero y 31 de marzo de 1944) declaró excluido de su ámbito regulador este sector. Posteriormente, la Ley de Bases de la Seguridad Social de 28 de diciembre de 1966 incluyó los servicios domésticos dentro de los Regímenes Especiales. Mediante el Decreto 2346/1969 (Régimen Especial de la Seguridad Social del Servicio Doméstico) el sector queda integrado en el sistema de la Seguridad Social, aunque con menor cotización y prestaciones. Será este decreto el que constituye la regulación básica que se ha mantenido en el tiempo.

Con la Ley de relaciones laborales de 8 de abril de 1976, el servicio doméstico pasa a ser considerado una relación laboral especial. Es importante señalar que en esta disposición se incide en la igualdad laboral del hombre y la mujer inclusive en la percepción de su remuneración, independientemente de su estado civil. Hasta entonces había existido una diferenciación clara, incluso reconocida por ley, entre los sueldos de hombres y mujeres y la posibilidad de establecer relaciones laborales por parte de las mujeres. Sin embargo, no es hasta 1985 cuando las relaciones laborales de las trabajadoras domésticas comienzan a considerarse laborales (Real Decreto 1424/1985) y

\footnotetext{
${ }^{54}$ SANTOS Y VALENCIA, 2011.

${ }^{55}$ LEBRUSÁN Y CÁCERES, 2017.
} 
se pone fin a la jurisdicción meramente civil del sector. Pasarán 26 años hasta que vuelva a legislarse sobre este sector históricamente desfavorecido (Real Decreto 1620/2011). Este último decreto supone un avance, en tanto que se produce el reconocimiento de la necesidad y el acercamiento a otros sectores laborales, creándose el Régimen especial de las empleadas de hogar.

Ese mismo año, la Ley 27/2011, de 1 de agosto, sobre actualización, adecuación y modernización del sistema de Seguridad Social integra el Régimen Especial de la Seguridad Social de los Empleados de Hogar en el Régimen General de la Seguridad Social, que supone un avance frente a diversas cuestiones ${ }^{56}$. Sólo un año más tarde se publicó el Real Decreto-Ley 29/2012, de 28 de diciembre, de mejora de gestión y protección social en el Sistema Especial para Empleados de Hogar y otras medidas de carácter económico y social. Estas nuevas medidas se centran en las condiciones de afiliación para las trabajadoras por horas y en los tramos de cotización de todas las trabajadoras del hogar, modificando la obligatoriedad de alta por el empleador en el caso de trabajar menos de 60 horas mensuales y suponiendo así un retroceso en el acceso a derechos laborales para muchas trabajadoras del hogar. Esto tiene una gran importancia de cara a la generación de derechos propios asociados a la historia laboral.

Asimismo, y a pesar de la gran responsabilidad que implican los cuidados (siendo incluso imprescindibles, vitales) el desempeño de los cuidados enmarcado en el mercado laboral continua siendo inferior en derechos a otros sectores como claro reflejo de su menor valorización social. Entre estas diferencias, existe un menor control y protección del sector, con ausencia de inspecciones laborales y de prevención de riesgos laborales, menores derechos sociales (como la no prestación por desempleo), un desplazamiento de la responsabilidad (la de estar al corriente de la seguridad social para las trabajadoras de menos de 60 horas) y en general una mayor desprotección laboral (con el despido por desistimiento) además de la permisión de condiciones abusivas ${ }^{57}$ (similares a las de otros del sector servicios) que paradójicamente estarían poniendo en riesgo la propia calidad de los cuidados emitidos. Para proteger a la persona que recibe los cuidados es necesario proteger a quien los ejerce.

En definitiva, la situación de estas trabajadoras (en femenino, ya que aproximadamente el 90\% de las personas que trabajan en servicio doméstico en España son mujeres) se caracteriza por una situación de desventaja frente a los empleados y empleadas del Régimen General, destacando la no posibilidad de poder cobrar la prestación por desempleo $^{58}$. Esto no deja de ilustrar esa desvalorización social que asume que las labores del cuidado, incluso cuando forman parte del empleo, pertenecen a otra esfera diferenciada de la productiva.

\footnotetext{
${ }^{56}$ Véase un análisis detallado de la normativa en LEBRUSÁN Y CÁCERES, 2017.

${ }^{57} \mathrm{Al}$ respecto, véase LEBRUSÁN y CÁCERES, 2017.

${ }^{58}$ LEBRUSÁN Y CÁCERES, 2017.
} 
Este desinterés normativo y desigualdad asociada puede leerse de dos formas: por una parte, se constata el desinterés social sobre este sector laboral. Por otra parte, las labores de cuidado, desplazada la responsabilidad del nivel social al nivel individual, tampoco se consideran lo suficientemente importantes como para favorecer la estabilidad de las trabajadoras que las realizan. Es decir, si bien los hogares encuentran en el servicio de hogar una estrategia decisiva para la continuidad de los cuidados, ni las políticas públicas la han considerado una prioridad ni el Sistema de bienestar ha ofrecido una alternativa $^{59}$. La desvalorización social del cuidado pasa de la propia labor a quien la lleva a cabo, incluso cuando queda sujeto a un contrato laboral.

A esta desvalorización en lo normativo y lo social, se añade la propia vulnerabilidad de quienes desempeñan estas labores. Al perfil de trabajo feminizado y minusvalorado, en la que se entrecruza la variable clase social, se añade en los últimos años la del origen ${ }^{60}$. La literatura académica ${ }^{61}$ señala un fuerte vínculo entre la demanda de trabajadoras para el servicio doméstico y la llegada de flujos migratorios feminizados que se convierten en mano de obra. Se produce una transferencia étnica, pero no de género, de modo que continúan siendo mujeres quienes realizan las tareas del hogar y los cuidados.

El de los cuidados, incluso cuando se considera empleo, continúa siendo un sector asociado a la vulnerabilidad y la desvalorización social, olvidando que sin cuidados no es posible la continuidad de la propia sociedad. Indudablemente, la primera dificultad para el reconocimiento de las labores de cuidados radica en el lugar de desempeño de la labor, que es el hogar (esfera privada). Las propias laborales desempeñadas quedan desprestigiadas; tradicionalmente se identifican como algo “que se hace por amor" por ello, de menor valor.

\section{REFLEXIONES FINALES}

El cuidado, considerado una cuestión privada, es fundamental para el sostenimiento de la vida y para la continuidad de la sociedad. Para que unas personas puedan trabajar (esfera productiva) otras deben dedicarse a la tarea del cuidado. Sin embargo, y a pesar de su importancia, las labores de cuidado quedan invisibilizadas y su responsabilidad, que debiera ser social, desplazada a la esfera individual.

Entre los motivos para su desvalorización, hay dos cuestiones que cabría señalar: la primera radica en el lugar de desempeño de la labor, que es el hogar y por lo tanto pertenece a la esfera privada, oculta, y en el que lo normativo tiene un menor alcance. La segunda cuestión sería el propio tipo de laborales desempeñadas: las del cuidado. Estas labores se identifican como algo “que se hace por amor" y libres de coste, lo que

\footnotetext{
${ }^{59}$ GARCÍA, SANTOS Y VALENCIA, 2014.

${ }^{60}$ La constatable etnificación se explica por la interconexión de las dinámicas económicas globales y locales (DÍAZ GORFINKIEL Y FERNÁNDEZ LÓPEZ, 2016).

${ }^{61}$ OSO, 1998; ESCRIVÁ, 2000; PARELLA, 2003; JULIANO, 2000 Y GREGORIO, 1998.
} 
paradójicamente, hace disminuir su valorización social. Esto contribuye a un constatable desinterés político y normativo tanto en lo que refiere a los cuidados formales como a los informales.

En una clara situación de inequidad, para dar respuesta a una demanda continua, se ha conformado socialmente la concepción de un sistema de provisión de cuidados informales y privados que quedan asumidos por la mujer. Esto, como hemos señalado, no implica un reconocimiento social ni de derechos.

Este sistema basado en la inequidad de género, queda alterado a tenor de diversos procesos propios de la sociedad actual: la normalización de la incorporación de la mujer al mercado laboral y un cambio de comportamiento (ya no deja el trabajo tras tener hijos o casarse); una mayor esperanza de vida, que hace que un mayor número de personas necesite cuidados durante más tiempo, pero también una diversificación del tipo de los mismos; un cambio en las pautas de convivencia e incluso en las relaciones familiares que dificulta la implementación de ciertas estrategias privadas; cierto desequilibrio demográfico según el cual cohortes de menor tamaños han de cuidar a un número mayor de personas (menor número de potenciales cuidadoras para una mayor demanda) además de una mayor exigencia en tiempo desde el mercado laboral que imposibilita la conciliación.

La respuesta por parte del Estado de bienestar es tibia, limitada e insuficiente: pese a la introducción de la Ley de Dependencia y el desarrollo de los servicios sociales u otras medidas orientadas al cuidado de la infancia, ni los poderes públicos ni las organizaciones privadas procuran una respuesta suficientemente satisfactoria a la demanda de cuidados. Es verdad que el Estado se amplía, cumple más funciones y se universaliza, pero también es cierto que se configura como un modelo en todo caso asistencial y puntual, pero no como la pata del Sistema de bienestar que la sociedad actual necesita que sea.

Lo anterior implica que sigan siendo las familias y especialmente las mujeres quienes continúen asumiendo el trabajo de cuidados, bien con un gran coste personal (doble jornada, que en el caso del cuidado a personas dependientes bien podría considerarse una triple jornada) o bien externalizándolo.

Como estrategia privada, una de las que más aumenta es la contratación de servicio doméstico, que pasa a ser decisiva en la organización doméstica familiar. Sin embargo, el Estado tampoco responde a esta dinámica fortaleciendo las relaciones laborales de las cuidadoras asalariadas.

Este, como hemos visto, es un sector laboral históricamente discriminado. A pesar de que a lo largo del tiempo se ha producido una mayor formalización del sector, quedan numerosos aspectos sobre los que legislar, destacando la situación del problema de 
invisibilidad, de acceso a derechos fundamentales y de derechos laborales, que postergan problemas sociales de cobertura universal.

Lo que denominamos aquí como la encrucijada de los cuidados es fruto de la inexistencia de un modelo de conciliación efectivo, con desastrosas consecuencias sobre quienes ejercen los cuidados, pero también sobre los potenciales demandantes de cuidados y las familias. Incluso cuando los cuidados son mercantilizados y externalizados fuera de la esfera familiar, continúan reflejando los efectos de la desvalorización social. Por otra parte, si bien el servicio doméstico sirve para hacer frente a las carencias de un Estado de bienestar reducido, es una falsa solución ante, primero, la necesidad de cuidados, y segundo, ante los problemas de conciliación familiar y laboral.

Lo anterior señala que, si el sector de los cuidados informales había sido tradicionalmente desfavorecido - no está ni ha estado reconocido de cara a la cotización, olvidando y silenciando esa contribución de las mujeres al Estado del bienestartampoco mejora su valoración o reconocimiento cuando pasa a considerarse empleo. Su importancia en la sociedad se minimiza, y esto hace que cuente con menor protección laboral que otros sectores. A pesar de su importancia y potencialidad como agente interviniente en la encrucijada señalada, dicho papel no se correlaciona con una mayor atención o facilidad de formalización de los cuidados por parte del Estado. Ni se facilita la contratación de empleadas domésticas ni se responde normativamente a las necesidades específicas de un sector que ya parte de una desvalorización social.

Si en el pasado se consideraba el esfuerzo de la mujer como pagado por una satisfacción basada en la relación afectiva, esa misma estructura se asume equivalente en una nueva relación de cuidados en la que no existe ningún tipo de lazo afectivo. Si en el pasado suponía una injusticia social que minusvaloraba el tiempo de la mujer, en la actualidad carece por completo de sentido.

Los vacíos que aún quedan presentes en la normativa resultan intolerables y suponen un riesgo para el bienestar de las trabajadoras, pero también para el de las personas que reciben los servicios y cuidados, y en definitiva y por ese motivo, para el conjunto de la sociedad.

En relación con lo anterior, quedan pendientes varias reflexiones: sobre el derecho a los cuidados y la responsabilidad social y estatal sobre los mismos; sobre el derecho referido brevemente a no cuidar; y más allá, sobre la calidad de los cuidados y cómo la legislación (o su ausencia y limitación) puede estar afectando a la calidad del cuidado tanto formal como informal ante la insuficiencia de apoyo y protección a quienes emiten los cuidados. 


\section{BIBLIOGRAFÍA}

ARANGO, J., DÍAZ, M., \& MOUALHI, D. (2013). Promover la integración de las trabajadoras y los trabajadores domésticos migrantes en España. Ginebra: Organización Internacional del Trabajo.

GIAMELLO, C. Y CASTRO, G.(2017): Nativos de la crisis: los niños de la llave. EDUCO, $2017 . \quad$ Disponible en: https://www.educo.org/Educo/media/Documentos/Prensa/Publicaciones/informe_nativo scrisis_educo_2017.pdf

GIAMELLO, C. Y CASTRO, G. (2018): Los otros niños de la llave. Cuando la precariedad se hace costumbre en España. $\operatorname{EDUCO}(2018)$. Disponible en: https://www.observatoriodelainfancia.es/oia/esp/documentos_ficha.aspx?id=5578

DÍAZ GORFINKIEL, M., \& FERNÁNDEZ LÓPEZ, C. (2016). Impacto de las reformas legislativas en el sector del empleo del hogar en España. Serie condiciones de trabajo y empleo, (82).

ELIZALDE SAN MIGUEL, B. (2018). ¿Femenino e informal? El modelo tradicional de cuidados a examen desde una perspectiva demográfica. Prisma Social: revista de investigación social, (21), 243-262.

ESCRIVÁ, A. (2000). The position and status of migrant women in Spain, en F. Anthias y G. Lazaridis (eds). Gender and migration in Southern Europe: women on the move. Oxford: Berg, pp. 199-225.

EZQUERRA, S. (2012). Acumulación por desposesión, género y crisis en el Estado español. REC.

FERNÁNDEZ CARRO, C. (2018). ¿Hacia la" desfamiliarización" del cuidado predilecto? Un análisis del contexto español (1997-2009). Reis: Revista española de investigaciones sociológicas, (164), 57-76.

FLAQUER, L., PFAU-EFFINGER, B., \& LEIRAS, A. A. (2014). El trabajo familiar de cuidado en el marco del Estado de bienestar. Cuadernos de Relaciones Laborales, 32(1), 11.

GARCÍA-CALVENTE, M. D. M., MATEO-RODRÍGUEZ, I., \& EGUIGUREN, A. P. (2004). El sistema informal de cuidados en clave de desigualdad. Gaceta Sanitaria, 18, 132-139. 
GARCÍA SÁINZ, C., SANTOS PÉREZ, M.L. \& VALENCIA OLIVERO, N. Y. (2014). La construcción social del mercado laboral doméstico en España a comienzos del siglo XXI. Cuadernos de Relaciones Laborales.

GREGORIO, C. (1998): Migración femenina. Su impacto en las relaciones de género. Madrid: Ed. Narcea.

HOLGADO GONZÁLEZ, M. (2019). Dependencia y derecho a los cuidados desde una perspectiva de género. Lex Social: Revista de Derechos Sociales, 9(1), 46-68.

JULIANO, D. (2000). Mujeres estructuralmente viajeras: estereotipos y estrategias. Papers, 60, 381-389.

LEBRUSÁN MURILLO, I. (2017). La vivienda en la vejez: problemas y estrategias para envejecer en sociedad (Doctoral dissertation, Universidad Complutense de Madrid).

LEBRUSÁN, I. Y CÁCERES, P. (2017). La mujer inmigrante en servicio doméstico. Análisis de la situación laboral e impacto de los cambios normativos (A.D. Los Molinos). Disponible en: http://www.admolinos.org/wpcontent/uploads/2017/07/ESTUDIO-MUJER-MIGRANTE-EN-SERVICIODOMESTICO.pdf

LEBRUSÁN, I. CÁCERES, P. \& BREY, E. (2019, aceptado, en proceso de publicación): El servicio doméstico como vía de acceso a la regularización administrativa en Madrid. Anuario CIDOB de la Inmigración 2019.

LEÓN, M. (2013). A real job? Regulating household work: The case of Spain. European Journal of Women's Studies, 20(2), 170-188.

MARTÍNEZ BUJÁN, R. (2014). Los modelos territoriales de organización social del cuidado a personas mayores en hogares. Revista Española de Investigaciones Sociológicas, 145, 99-126. DOI: 10.5477/cis/reis.145.99

MEIL, G. (2005). La protección social de la familia: situación actual y tendencias en la Unión Europea. En: Políticas hacia las familias, protección e inclusión sociales-LC/L. 2373-P-2005-p. 19-28.

MOLPECERES ÁLVAREZ, L. (2012). Situación laboral de las mujeres inmigrantes en España. Cuadernos de Relaciones Laborales, 30(1), 91.

MORENO FERNÁNDEZ, L., \& MARI-KLOSE, P. (2016). Bienestar mediterráneo: trayectorias y retos de un régimen en transición. Los Estados de bienestar en la 
encrucijada. En: Políticas sociales en perspectiva comparada, $2^{\mathrm{a}}$ ed. aumentada, Madrid: Tecnos, 139-160.

MORENO FUENTES, F. J. \& BRUQUETAS CALLEJO, M. (2011). Inmigración y Estado de bienestar en España, Obra Social La Caixa. Colección de Estudios Sociales, (31).

NAVARRO, V. (2004) El Estado de bienestar en España (Madrid, Tecnos).

NAVARRO, V., \& QUIROGA, Á. (2004). Políticas de Estado de bienestar para la equidad. Gaceta Sanitaria, 18(4), 147-157.

NOGUEIRA DOMÍNGUEZ, J. \& ZALAKAIN, J. (2015). La discriminación múltiple de las mujeres extranjeras que trabajan en servicios domésticos y de cuidados a dependientes en Euskadi. Zerbitzuan: Gizarte zerbitzuetarako aldizkaria= Revista de servicios sociales, (60), 143-162.

OROZCO, A. (2007). Cadenas globales de cuidado. Serie: Género, Migración y Desarrollo. Documento de trabajo $n^{\circ} 2$.

OROZCO, A. P., PAIEWONSKY, D., \& GARCÍA, M. (2008). Cruzando fronteras: Migración y desarrollo desde una perspectiva de género. Instituto de la Mujer.

OSO, L. (1998). La migración hacia España de mujeres jefas de hogar. Madrid: IMU

OSO, L., \& PARELLA, S. (2012). Inmigración, género y mercado de trabajo: una panorámica de la investigación sobre la inserción laboral de las mujeres inmigrantes en España. Cuadernos de Relaciones Laborales, 30(1), 11-44.

OTXOA CRESPO, I. (2011). La Seguridad Social del empleo doméstico: evolución y perspectivas. (Doctoral dissertation, Universidad del País Vasco)

PADILLA SÁNCHEZ, I; ORDAZ SANZ, J.A; GUERRERO CASAS, F.M (2013) El servicio doméstico en España: un estudio econométrico (congreso).

PARELLA, S. (2003). Mujer, inmigrante y trabajadora. La triple discriminación. Barcelona: Anthropos.

PAZOS MORÁN, M. (2013). Desiguales por ley: las políticas públicas contra la igualdad de género. Los Libros de la Catarata.

PENALVA, A. S. (2019). Las cargas familiares: un clásico motivo de discriminación en el ámbito de las relaciones laborales. In Retos en materia de igualdad de género en el Siglo XXI (pp. 289-302). Dykinson. 
ROBLES SILVA, L. (2003). Doble o triple jornada: el cuidado a enfermos crónicos. Estudios del hombre, (17), 75-99.

SABATER FERNÁNDEZ, M. C. (2014). La interacción trabajo-familia. La mujer y la dificultad de la conciliación laboral. Lan harremanak: Revista de relaciones laborales, (30), 163-198.

SANTOS, M. L., \& VALENCIA, N. (2011). Inmigrantes en el servicio doméstico. (C. García, Ed.) Madrid: Talasa Ediciones.

SERRANO, A., MORENO, F. \& CRESPO, E. (2001). La experiencia subjetiva del trabajo en una sociedad en transformación. En Trabajo, individuo y sociedad: perspectivas psicosociológicas sobre el futuro del trabajo (pp. 49-64). Ediciones Pirámide.

TOBÍO, C. (2001). Nuevas formas familiares, viejas formas urbanas. Boletín CF+ S, (22).

TOBÍO, C. (2013). Estado y familia en el cuidado de las personas: sustitución o complemento. Cuadernos de relaciones laborales, 31(1), 17-38.

TOBÍO, C., AGULLÓ TOMÁS, M. S., GÓMEZ, M.V. Y MARTÍN PALOMO, M.T. (2010). El cuidado de las personas. Barcelona, España: La Caixa.

TORNS, T. (2011). Conciliación de la vida laboral y familiar o corresponsabilidad: ¿el mismo discurso?. RIDEG: revista interdisciplinar de estudios de género, (1), 0005-13.

VALIENTE FERNÁNDEZ, C. (1997). ¿Algo más que" ganadores del pan"?: El papel de los hombres en el ámbito familiar en España (1975-1996). Reis, 221-243. 